\title{
Propagation at mmW Band in Metropolitan Railway Tunnels
}

\author{
Ana González-Plaza $\mathbb{D D}^{1}{ }^{1}$ César Calvo-Ramírez, ${ }^{1}$ César Briso-Rodríguez ${ }^{(\mathbb{D})}{ }^{1}$ \\ Juan Moreno García-Loygorri, ${ }^{1}$ David Oliva, ${ }^{2}$ and José I. Alonso ${ }^{2}$ \\ ${ }^{1}$ Departamento de Teoría de la Señal y Comunicaciones, ETSIS de Telecomunicación, \\ Universidad Politécnica de Madrid, Madrid, Spain \\ ${ }^{2}$ Departamento de SSR, ETSI de Telecomunicación, Universidad Politécnica de Madrid, Madrid, Spain
}

Correspondence should be addressed to Ana González-Plaza; gonzalezplaza.ana@gmail.com

Received 3 November 2017; Revised 17 January 2018; Accepted 14 February 2018; Published 26 March 2018

Academic Editor: Eva Antonino-Daviu

Copyright (C) 2018 Ana González-Plaza et al. This is an open access article distributed under the Creative Commons Attribution License, which permits unrestricted use, distribution, and reproduction in any medium, provided the original work is properly cited.

\begin{abstract}
The next generation of mobile communications, 5G, will provide a wideband network based on microwave and millimeter-wave $(\mathrm{mmW})$ communication radio links with the goal of fulfilling the strict and severe requirements of the future test cases. In particular, this paper research is focused on $\mathrm{mmW}$ bands in metropolitan railway tunnels. For that purpose, a propagation measurement campaign was performed at $24 \mathrm{GHz}$ band in a passenger train on a realist subway environment, and these results were combined with simulations ad hoc for tunnels and a theoretical modal propagation model. A narrowband and a wideband study have been conducted with the aim of obtaining the path loss, fading, power-delay profile, and angle of arrival, taking into consideration horizontal and vertical polarization in the receiving and transmitting antennas. This validation can be used to design and deploy wideband mobile communication networks at $\mathrm{mmW}$ bands in railway scenarios.
\end{abstract}

\section{Introduction}

The anticipated increase in demand for data traffic is one of the key requirements of the upcoming years in mobile communication technologies. The fifth generation (5G) aims to provide considerable broader bandwidths and a network capable of offering a flexible technology for very different cases studies [1].

In this context, $5 \mathrm{G}$ could be the most disruptive technology in railways since it can allow the addition of new services and applications that make railway a more efficient, safer, and profitable transportation option [2].

However, there are important challenges to overcome: The first one is to provide a wideband service for the plenty of passengers who will demand a large capacity. The second one is the critical signalling control applications which will require very high quality of service and moderate data rate to allow real-time applications such as high definition video for automatic driving and surveillance $[3,4]$.

In the case of signalling, current railway mobile communication systems such as GSM-R are not able to provide such ambitious features [5]. For this reason, LTE-R and future $5 \mathrm{G}$ are being examined as new railway communication technologies. In the latter, $3 \mathrm{GPP}$ new radio (NR) system for $5 \mathrm{G}$ proposes a large spectrum solution from bands under $6 \mathrm{GHz}$ to $100 \mathrm{GHz}$ [6]. Among all, millimeter-wave (mmW) bands, particularly from 24 to $33 \mathrm{GHz}$, are considered as a suitable option to establish mobile communication services in high-speed scenarios. This band has been chosen because it is an industrial scientific and medical band (ISM), which can be used for railway communications. In fact, 28 and $37 \mathrm{GHz}$ have been already licensed in the USA and $26 \mathrm{GHz}$ band in China and Europe [7].

The study of electromagnetic field propagation in the different specific scenarios of the railway environment is a key prerequisite in order to succeed in the design and installation of these mobile communication systems. In this sense, $\mathrm{mmW}$ communications in tunnels offer some interesting features over lower frequency bands for communications in tunnels [8].

(i) Electromagnetic discharges of catenary pulses, usual in tunnels, have little influence on millimeter-wave propagation. 
(ii) Losses produced by reflection and refraction of electromagnetic waves at mmWs are relatively small.

(iii) Very wide bandwidth is available.

(iv) Latency reduction is achieved.

Nevertheless, an assessment of the propagation channel is a mandatory requirement to ensure reliable communication links. For that reason, although there is already some literature on $\mathrm{mmW}$ measurements in tunnels at $30 \mathrm{GHz}$ [9], $38 \mathrm{GHz}$ [10], $60 \mathrm{GHz}$ [11], and $240 \mathrm{GHz}$ [12] bands, there are no measurements in a realistic railway environment.

This paper focused its research on the propagation at $24 \mathrm{GHz}$ frequency band in realistic metropolitan railway tunnels. For that purpose, three methods have been applied. Firstly, a modal propagation model has been described and put into practice; then, a ray tracing (RT) simulator developed specially for propagation in tunnels has been employed; and, finally, measurements in a passenger train on a realist subway environment have been performed.

The goal of this study is to present an accurate path loss model at $24 \mathrm{GHz}$ directly applicable to railway communication systems and to show meaningful results for wideband simulations to assess the possibility of using $\mathrm{mmW}$ for communication systems in metropolitan railway tunnels.

This analysis presents some results regarding the path loss and the fading, power-delay profile (PDP), and angle of arrival (AoA) along a tunnel, taking into account horizontal and vertical polarization in the receiving and transmitting antennas.

This document is organized as follows: firstly, in Section 2, a theoretical attenuation modal model and the 3D RT technique used by the simulator are explained; secondly, Section 3 describes Raylway simulation tool and the parameters configured for this experiment in the real environment and in the simulator; afterwards, in Section 4, all the results are enumerated: path loss model and $K$ factor in Section 4.1 and PDP and AoA in Section 4.2; finally, Section 5 presents the conclusion obtained from all this work.

\section{Modeling and Simulation of Guided Propagation in Tunnels}

In the past, the modal theory has been widely applied to study the propagation in tunnels due to it provides suitable results. However, this method does not take into account the effect of arbitrary geometries in walls, roughness, discontinuities, among others. For that reason, nowadays, RT methods are employed to obtain more accurate propagation models [13].

2.1. Modal Analysis. Modal analysis is a classical technique for studying propagation channel in tunnels where there is waveguide effect. It is based on the concept that the signal attenuation in this kind of scenarios depends on the electromagnetic modes propagating within the tunnel. As the cross-section area of the tunnel is quite large in comparison to the signal wavelength, there are many modes propagating through this channel. Nevertheless, the modes suffer gradual extinction as they travel along the environment up to the breakpoint, $Z_{\mathrm{NF}}$, where the modes are sufficiently attenuated and it can be considered that only the first mode propagates.

$$
Z_{\mathrm{NF}} \approx \max \left(\frac{a^{2}}{\lambda}, \frac{b^{2}}{\lambda}\right),
$$

where $a$ and $b$ are the horizontal and vertical dimension of the tunnel, respectively, and $\lambda$ is the signal wavelength.

These two regions are defined as near-field and far-field regions. On the one hand, the near-field region represents the zone where the field amplitude suffers strong fading and fast losses obtained from the contribution of many rays propagating from different grazing angles with high losses. On the other hand, the far-field region is where the relation between the first mode and the rest is high enough to neglect the effect of the other modes.

As [14] explains in detail, the energy contained in the transmitted signal propagating through the tunnel is divided into several modes. Each of them is attenuated following different decay. The overall path loss can be simplified by considering that each mode has an independent power from the others. Therefore, the total received power is approximately the sum of all modes' power. This supposition is based on the fact that modes are closely orthogonal.

Assuming this situation, the loss expression of each mode can be approximated for horizontal and vertical polarization, $\alpha(m, n)^{h, \nu}$, considering the tunnel as a rectangular waveguide.

$$
\begin{gathered}
\alpha(m, n)^{\nu}=4.343 \lambda^{2}\left(\frac{m^{2}}{a^{3} \sqrt{\varepsilon_{r_{1}}-1}}+\frac{n^{2} \varepsilon_{r_{2}}}{b^{3} \sqrt{\varepsilon_{r_{2}}-1}}\right), \\
\alpha(m, n)^{h}=4.343 \lambda^{2}\left(\frac{m^{2} \varepsilon_{r 1}}{a^{3} \sqrt{\varepsilon_{r_{1}}-1}}+\frac{n^{2}}{b^{3} \sqrt{\varepsilon_{r_{2}}-1}}\right),
\end{gathered}
$$

where $\alpha(m, n)^{\nu, h}$ are the attenuation of each mode in $\mathrm{dB} / \mathrm{km}$ in vertical $(\nu)$ and horizontal $(h)$ polarization for the $m, n$th mode; $\varepsilon_{r_{1}, r_{2}}$ are the relative permittivity of the vertical and horizontal walls, respectively. This model can be also applied to arched tunnels by adjusting the cross-section dimensions [15].

As the equations in (2) show, tunnels dimensions are directly related to the attenuation that every mode suffers. Thus, the greater the tunnel cross-section is, the smaller the attenuation experienced by the modes is. This is because losses are inversely proportional to the cube of tunnels height and width.

With this propagation constant, the overall attenuation for both polarization directions, $L_{m n}^{v, h}$, at a certain distance, $(z)$, considering losses as the rms contribution of modes for both polarization directions is the following:

$$
\begin{aligned}
& L_{m n}^{v}(z)=10 \log _{10}\left[\sum_{n=1}^{M} \sum_{m=1}^{N} 10^{\left(1 \log _{10}(z)+\alpha(m, n)^{\nu}\right) / 10}\right], \\
& L_{m n}^{h}(z)=10 \log _{10}\left[\sum_{n=1}^{M} \sum_{m=1}^{N} 10^{\left(10 \log _{10}(z)+\alpha(m, n)^{h}\right) / 10}\right],
\end{aligned}
$$

where $M$ and $N$ are the last modes taken into account. 


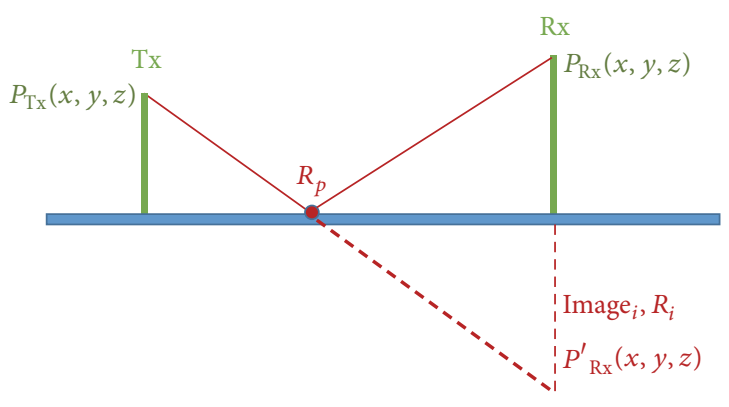

FIgURE 1: The image method. $R_{i}$ with respect to the ground plane.

In addition to this, losses are directly proportional to the square of the order of the modes, so that higher modes experience stronger attenuation. In fact, we can consider that, from a certain distance, all high order modes have been extinguished and only the fundamentals remain.

However, the modal propagation model does not take into account tilt losses, which are more important in semicircular tunnels and must be adjusted carefully for each tunnel, or wall roughness in tunnels, which might produce scattering of the signal. That is why simulations using RT technique are performed to obtain more accurate results.

2.2. Ray Tracing Technique. This simulator uses the novel technique of 3D ray tracing which is based on a geometrical optics method to compute the propagation channel of the modeled environment. It is an accurate and versatile propagation prediction tool for different kind of scenarios [16]. It uses a deterministic channel model which requires an exact knowledge of the geometrical and electromagnetic description of the environment. For that reason, the accuracy of RT comes up with a high computational complexity, which directly scales with the number of propagation paths considered. Nevertheless, it has great advantages as, on the one hand, it allows really detailed modeling, and, on the other hand, with this technique, it is possible to calculate wideband information such as PDP or AoA of the rays.

Tunnels are modeled as a triangular solid mesh made of different materials whose dielectric properties are defined according to the carrier frequency. As this is a $3 \mathrm{D}$ RT, reflections on the ceiling and ground are taken into account. This provides more accurate prediction results compared with conventional 2D models.

Using the images method, reflections are calculated in order to obtain the propagation paths. This method consists of basically converting an electric field into an equivalent one easier to compute. In certain cases, it is possible to replace a conductor with one or more point loads. Thus, conductor surfaces are replaced by equipotential surfaces with equivalent potentials.

As Figure 1 shows, having the location of $\mathrm{Tx}$ and $\mathrm{Rx}$, the reflection point, $R p$, coincides with the intersection between the obstacle and the segment joining the Tx with the image of the receiver, $R_{i}$. Therefore, the ray follows a straight line from $\mathrm{Tx}$ to $R p$ and, then, another straight line from $R p$ to $\mathrm{Rx}$. It is possible to extend this method to obtain the paths with multiple reflections. Finally, the received signal, $E_{r}$, is the sum of all the rays that arrived at $\mathrm{Rx}$, as follows:

$$
\begin{aligned}
E_{r} & =\left|\sum_{i} E_{i}\left(d=d_{\mathrm{rx}}\right)\right|, \\
E_{i}(d) & =E_{0} \rho_{\mathrm{tx}} \rho_{\mathrm{rx}} L_{i}(d) \prod_{p}^{N}\left[\Gamma\left(\phi_{p i}\right) T\left(\phi_{p i}\right)\right] e^{-j 2 \pi d / \lambda},
\end{aligned}
$$

where $E_{i}(d)$ is the $i$ th ray electric field along the distance; $d_{\mathrm{rx}}$ is the distance between the transmitter and the receiver; $E_{0}$ is the amplitude of the electromagnetic field; $\rho_{\mathrm{tx}}$ and $\rho_{\mathrm{rx}}$ are the radiation pattern of the transmitter and receiver antenna, respectively; $L_{i}(d)$ is the path losses for $i$ th ray component at a distance $d ; \Gamma\left(\phi_{j i}\right)$ and $T\left(\phi_{p i}\right)$ are the reflection and transmission coefficient of the pth reflection of the $i$ th component, respectively; $N$ is the maximum reflections taken into account; and $e^{-j 2 \pi d / \lambda}$ is the phase factor due to the travelled distance.

Besides, this simulator calculates the power-delay profile which is a temporal representation of the pulse widening. The instantaneous impulse response [17], $h(t, \tau)$, is function of the time, $t$, and the propagation delay, $\tau$. The power-delay profile, $\operatorname{PDP}(\tau)$, is described by (5) when the channel satisfies the wide sense stationary uncorrelated scattering assumption [18].

$$
\operatorname{PDP}(\tau)=\left\langle|h(\tau, t)|^{2}\right\rangle .
$$

Lastly, the angle of arrival is a method for obtaining the propagation direction of a radio-frequency wave incident on a virtual array antenna. The direction is determined by measuring the received signal in each of the elements of the virtual array. This difference in phase is measured by receiver nodes $R, A 1$, as follows:

$$
\Delta \varphi=\frac{2 \pi}{\lambda}\left(d_{R A_{2}}+d_{A_{1} A_{2}}+d_{T A_{1}}-d_{T R}\right),
$$

where $R$ is the receiver position with its separated virtual elements $A_{1}$ and $A_{2}$ and $T$ represents the position of the transmitter.

Hence, the use of 3D-RT technique allows the detailed study of the behavior of wideband channel.

\section{Simulation and Measurement Set-Up}

3.1. Simulation Tool. Simulations are performed using Raylway: Radio Propagation Simulator for Tunnels tool, which calculates the radio signal propagation along different tunnel environments. The software, developed in Java and Matlab, allows the user to design and set up the tunnel in order to analyze the propagation in a specific environment. Correspondingly, Raylway software brings the received power, the power-delay profile, and the angle of arrival.

This simulator allows users to set up several parameters in order to obtain results according to the tunnel under study:

(i) tunnel: definition of the different cross-section types plus their shape and the longitudinal section and wall material properties; 




FIGURE 2: Simulator user interface with set-up parameters.

(ii) transmitter: initial fixed position, frequency, and transmitted power;

(iii) receiver: initial position, frequency, and distance travelled;

(iv) antennas: radiation pattern of each antenna, polarization, and relative position between them;

(v) simulation settings: number of simulation points, maximum number of each ray reflections, and possibility of enabling diffraction.

3.2. Simulation Set-Up. Raylway user interface is presented in Figure 2 where the set-up parameters are shown, in this case, for vertical polarization; however, except the polarization, the same values are maintained for the horizontal polarization. The resulting set-up simulation scenario is shown in Figure 4.

3.3. Measurement Set-Up. In this section, the measurement set-up in a realistic subway tunnel is explained in detail.

This experiment was conducted in a subway tunnel of Metro de Madrid. Figure 3(b) illustrates the scenario with its more important elements. The set-up parameters are enumerated in Table 1.

On the one hand, the transmitter (Tx) is situated in a fixed position between one station and the tunnel; on the other hand, the moving receiver $(\mathrm{Rx})$ module is located on the windshield of the train. The same antenna model is used in the Tx and Rx equipment. It is a horn antenna of Vector Telecom with model number VT260SGAH15 [19] which covers the frequency range from $21.7 \mathrm{GHz}$ to $33 \mathrm{GHz}$. Its radiation pattern provided at $23.96 \mathrm{GHz}$ is shown in Figure 5. The gain is a $15.2 \mathrm{dBi}$ and the half power beamwidth is $28.3^{\circ}$ in $E$-plane and $27.9^{\circ}$ in $H$-plane.

The tunnel has an arched type tunnel which can be approximately modeled as an equivalent rectangular crosssection since there is a slight adaptation of the dimensions,
TABLE 1: Set-up parameters.

\begin{tabular}{lc}
\hline Frequency & $24.2 \mathrm{GHz}$ \\
Transmitted power & $5.4 \mathrm{dBm}$ \\
Tx signal & Continuous wave \\
\hline Tx and Rx antenna & Horn antenna, $15.2 \mathrm{dBi}$ \\
HPBW antenna & $E$-plane $28.3^{\circ}$ \\
& $H$-plane $27.9^{\circ}$ \\
Antenna polarization & Horizontal and vertical \\
\hline Tunnel cross-section & Arched \\
Tunnel dimensions & $6.9 \mathrm{~m} \times 4.9 \mathrm{~m}$ \\
Train speed & $5 \mathrm{~km} / \mathrm{h}$ \\
\hline
\end{tabular}

according to [15]. To apply the modal propagation model described in Section 2.1, in this case, the dimensions are $6.9 \mathrm{~m}$ $\times 4.9 \mathrm{~m}$ with a radius of $2.2 \mathrm{~m}$, as Figure 3(a) shows. The assessed route follows a straight line of $350 \mathrm{~m}$ with a minor slope of $5.7 \%$. The point 0 represents where the Tx is situated.

This train moves along the railway up to $350 \mathrm{~m}$ at a constant speed of $5 \mathrm{~km} / \mathrm{h}$.

\section{Results}

In this section, both modal analyses and RT technique together with measurement conducted in a real metropolitan railway tunnel are applied with the aim of assessing the propagation in this particular environment at $24 \mathrm{GHz}$ band.

The analysis is focused on the path loss model, fastfading, power-delay profile, and angle of arrival.

4.1. Narrowband Analysis. This section provides a comparison between measurements, simulation, and modal propagation model for vertical and horizontal polarization. For that purpose, two experiments were performed: the first one with vertical polarization in both antennas and the second one with horizontal polarization. The train follows the exact same route in both experiments; likewise, two simulations were performed with the different polarization directions; and, finally, the theoretical modal losses are shown in order to compare these different approaches.

4.1.1. Path Loss Model. The attenuation suffered by a signal can be described through a path loss model defined by (7). The main parameters are derived from the following measurements conducted in a real environment:

$$
A(d)=A\left(d_{0}\right)+10 n \log \left(\frac{d}{d_{0}}\right)+\chi_{\sigma},
$$

where $A\left(d_{0}\right)$ is attenuation in a reference distance $d_{0}, n$ represents the loss exponent, and $\chi_{\sigma}$ denotes a zero-mean random variable of standard deviation $\sigma$ which follows a Gaussian distribution.

Figure 6 presents the received power of the empirical data, the simulation result, the theoretical modal model, and the resulting path loss model for the vertical, Figure 6(a), and horizontal, Figure 6(b), experiments, respectively. 




(a)

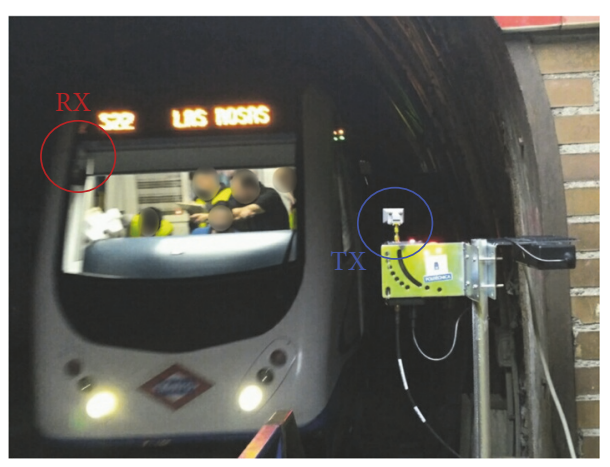

(b)

Figure 3: (a) Tunnel cross-section dimensions and shape. (b) Measurement scenario: fixed position of Tx at the beginning of the tunnel and moving $\mathrm{Rx}$ at the windshield of the train.

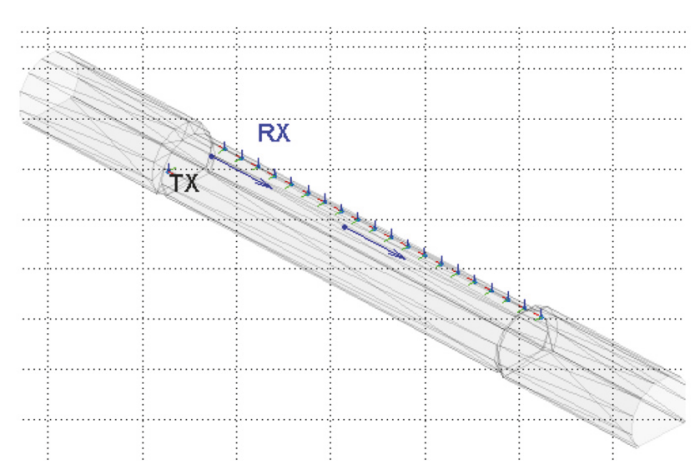

FIGURE 4: Simulation set-up scenario with fixed Tx (in black) and moving $\mathrm{Rx}$ (in blue).

Since the antennas are highly directive, as Figure 5 shows, the losses caused by the misalignment of the antenna are very high for distances lower than $20 \mathrm{~m}$. In addition to that, RT simulations have a strong dependency of the number of reflections for short distances. Hence, the simulation results are not accurate and the first $20 \mathrm{~m}$ has been removed from Figure 6.

In both experiments, the measurements expose a loss exponent, $n$, equal to 1.3 approximately, which is lower than the decay of the free space. Besides, as [20, 21] explains, regardless the type of tunnel, loss exponents are always lower than the free space. This is due to waveguide effect produced in tunnels.

The curve obtained from the modal analysis presents a similar attenuation since the loss exponents are 1 for vertical and 1.1 for horizontal polarization. This shows a significant effect of the modal propagation. Likewise, simulation results are slightly greater than the one acquired by the measurements since the loss exponent is 1.8 for vertical and 1.9 for horizontal polarization. These results are summarized in Table 2.

The differences between models may be produced because, on the one hand, in the modal model just the losses caused by the reflections are taken into account, and, on the other hand, in the case of the RT simulations, the maximum

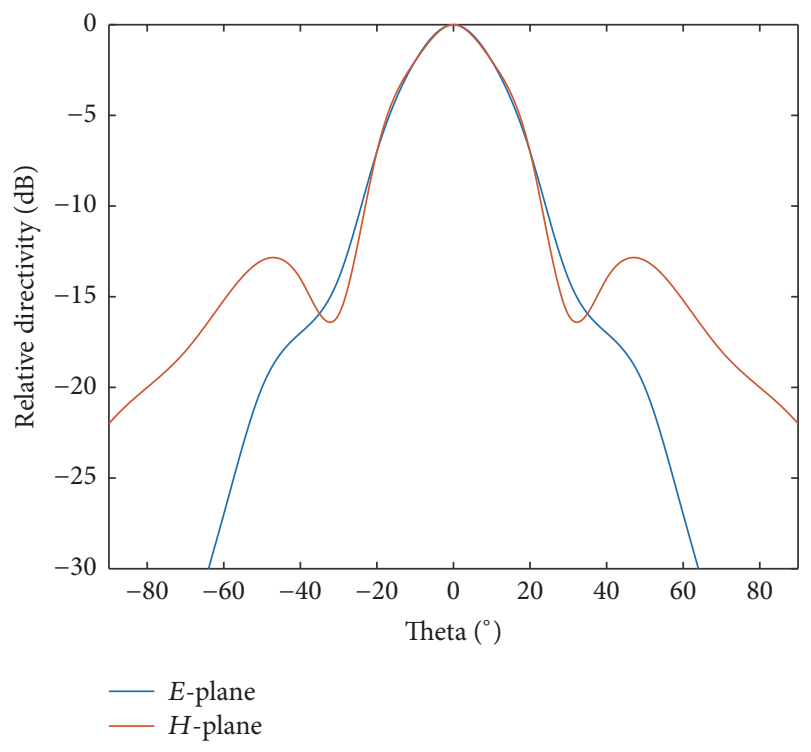

FIGURE 5: Horn antenna radiation pattern.

TABLE 2: Path loss model parameters at the vertical and horizontal polarization directions.

\begin{tabular}{lcc}
\hline & Vertical & Horizontal \\
\hline Ref. attenuation, $A\left(d_{0}\right)$ & $64.8 \mathrm{~dB}$ & $62.9 \mathrm{~dB}$ \\
\hline Measurement $n$ & 1.28 & 1.29 \\
Simulation $n$ & 1.8 & 1.9 \\
Modal propagation model $n$ & 1 & 1.1 \\
\hline Measurement $\chi_{\sigma}$ & $1.4 \mathrm{~dB}$ & $1.3 \mathrm{~dB}$ \\
Simulation $\chi_{\sigma}$ & $1.2 \mathrm{~dB}$ & $0.9 \mathrm{~dB}$ \\
\hline
\end{tabular}

The attenuation is at the reference point, $d_{0}$, of $20 \mathrm{~m}$. $A\left(d_{0}\right)$ is referred to the parameters in (7), as $n$ and $\chi_{\sigma}$.

number of reflections used is limited to six which seems to be not enough to calculate a more accurate model; nevertheless, after some distance, the results converge when the reflections are high enough, as Figure 7 shows. 

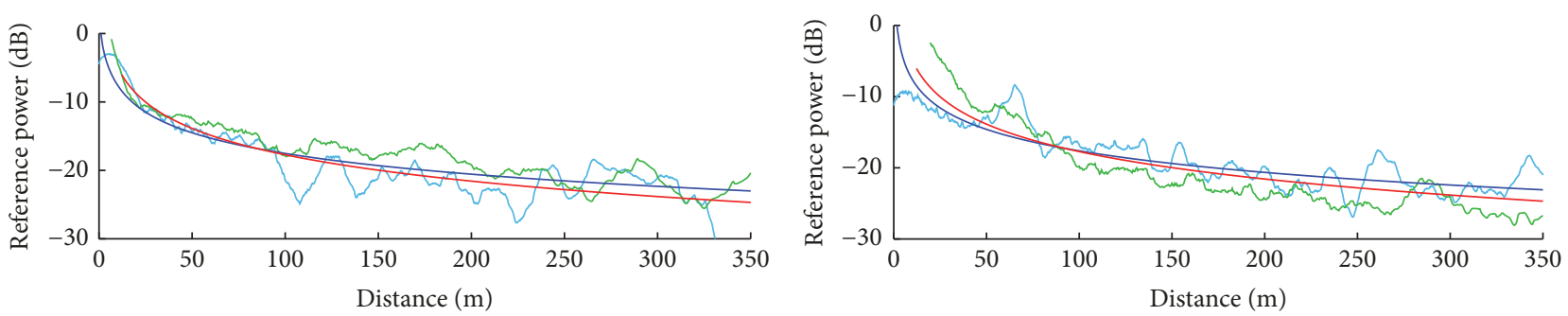

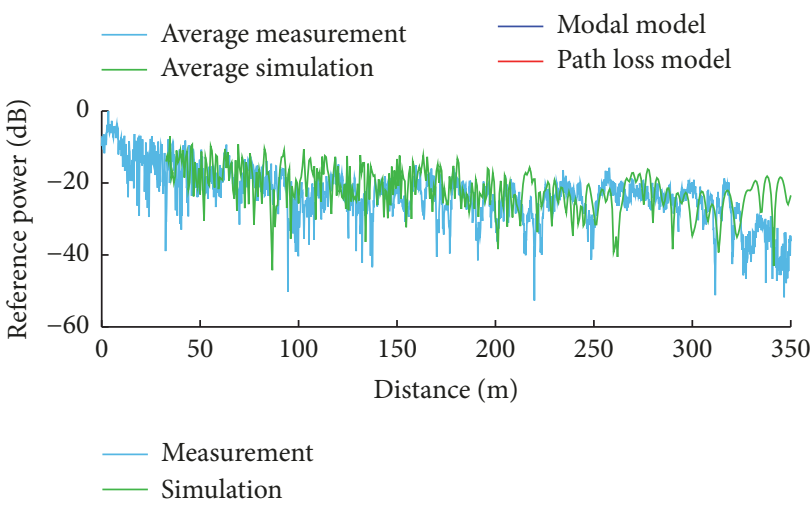

(a) Vertical polarization

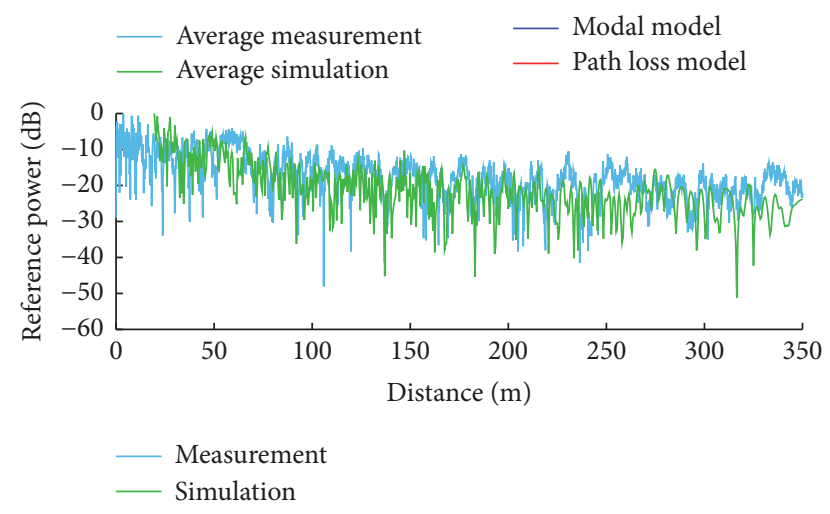

(b) Horizontal polarization

FIGURE 6: Comparison between measurements, simulation, and modal model for vertical and horizontal polarization.

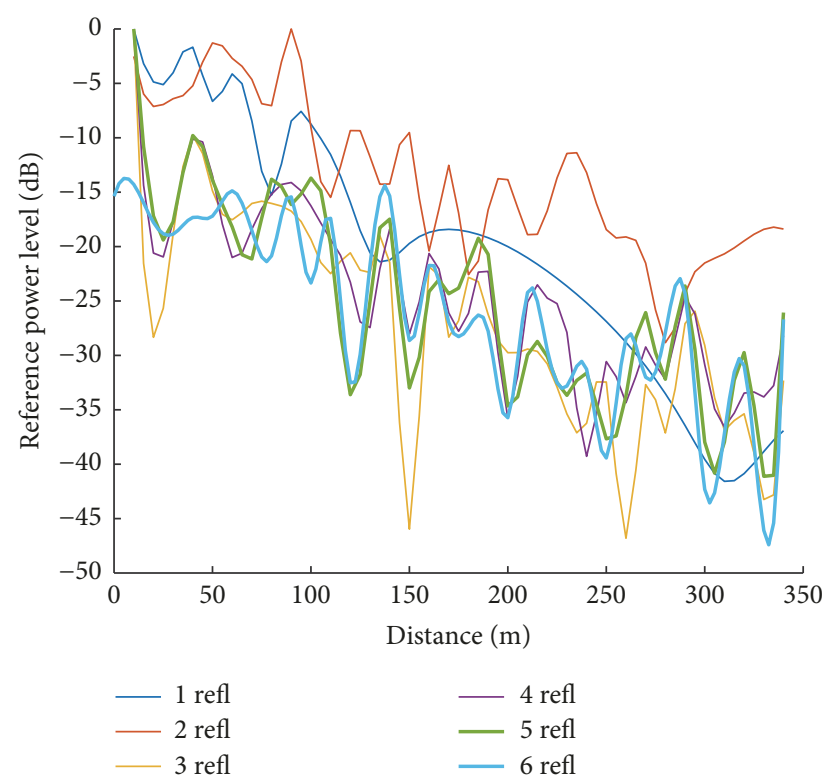

FIGURE 7: RT Simulation results for different number of each ray's reflections.

Nevertheless, in all the cases, the loss exponents are lower than those in the free space which show a clear waveguide effect. This is because the electromagnetic waves are repeatedly reflected in the tunnel's walls. As concrete is made of conductor materials (among others) the reflection coefficient is high. For that reason, the waves are confined to the dielectric by almost total internal reflection at its surface.
At mmW bands, a large number of modes are propagated through the tunnel. Particularly at $24.2 \mathrm{GHz}$, according to rectangular waveguide theory cut-off frequencies, modes up to $m$ and $n$ equal to 1246 and 1877, respectively, travel down the tunnel.

For that reason, the breakpoint, where most of them are sufficiently extinguished, is located at a few hundred meters in lower frequencies (from 1 to $6 \mathrm{GHz}$ ) [14]. However, at $24.2 \mathrm{GHz}$, it is at $8 \mathrm{~km}$ which means that theoretically the route is fully situated in the waveguide region.

The polarization seems to be independent of the loss exponent since it does not show a substantial difference in any of the cases. As Table 2 shows, this also holds true for the shadowing whose standard deviation is closely equal in both polarization directions.

The effect of the slope reveals an interesting difference in both polarization directions. There is a slope of $5.7 \%$. Thus, focusing on the average of the last $50 \mathrm{~m}$ where this slope has its major effect, there are greater losses in vertical polarization. This result is noticed in the simulations and in the measurements. It may be due to, firstly, the general misalignment of both antennas, which is analyzed in Section 4.2, and, secondly, the fact that this effect is more notable in vertical polarization since these antennas present higher directivity in elevation than in azimuth.

Regarding the reference attenuation, $A\left(d_{0}\right)$, there is a slight difference between polarization directions. In the case of horizontal polarization, there are $2 \mathrm{~dB}$ more than in vertical. This is related to the tunnel dimensions that are directly connected to the effect of polarization in the antennas. When tunnel presents larger width than its height, higher reflection coefficients appear in vertical polarization. Lower attenuation 


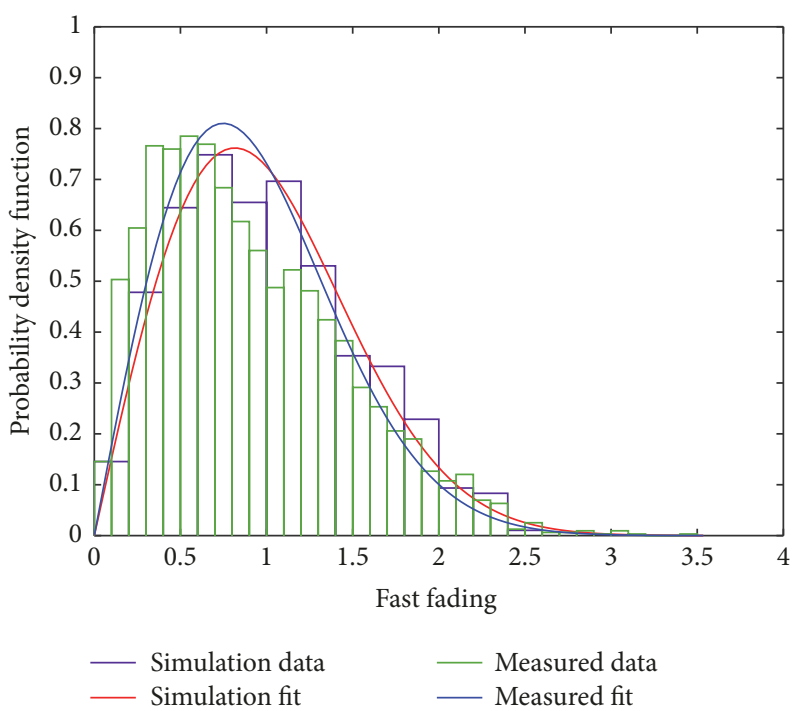

(a) Vertical polarization

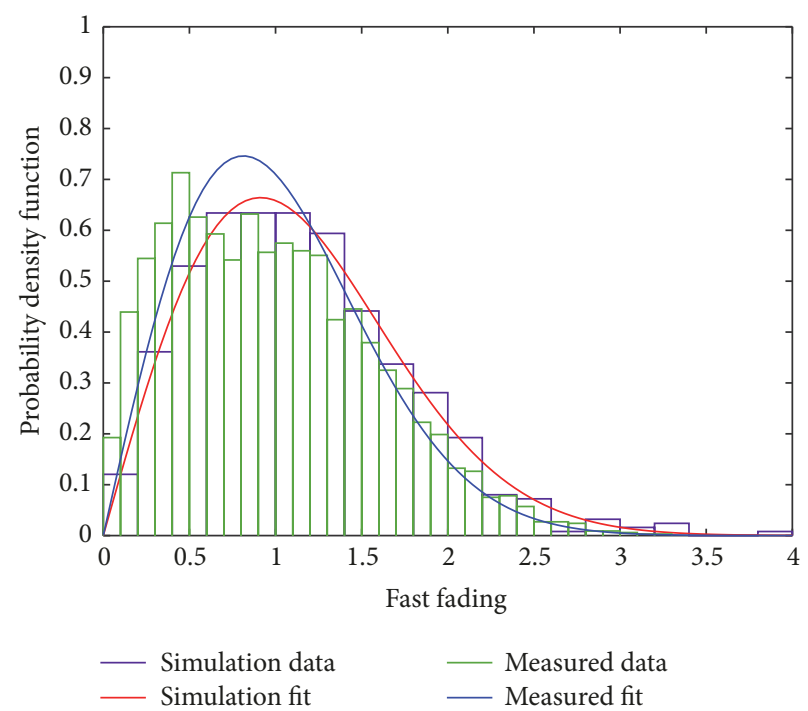

(b) Horizontal polarization

Figure 8: Comparison between measurements and simulation of the fast-fading probability density function.

comes out for the case of horizontal polarization. This effect is more significant in short distances. As [22] explains, in tunnels where there is a perfectly square cross-section, the result of vertical and horizontal polarization will be exactly the same.

4.1.2. Fast Fading. In this section, an analysis of the fast fading is shown. The results for vertical and horizontal polarization are described in Figure 8. A comparison between the measurements and simulations are made.

Rician distribution, $P_{r}(r)$, is a statistical model which better describes the fast-fading effect in an environment where there are LoS conditions.

$$
P_{r}(r)=\frac{r}{\sigma^{2}} e^{\left(r^{2}+A^{2}\right) / 2 \sigma^{2}} I_{0} \frac{r A}{\sigma^{2}},
$$

where $r$ is a random variable; $\sigma$ is the standard deviation; and $K$ factor, represented in (9), is the relation between LoS component, $A$, and multipath component.

$$
K=10 \log \left(\frac{A^{2}}{2 \sigma^{2}}\right) .
$$

At this frequency, as Table 3 enumerates, the channel varies considerably fast independently of the experiment since the wavelength is small, $12.4 \mathrm{~mm}$.

As Figure 6 shows, there are peaks up to $25 \mathrm{~dB}$ of attenuation in both cases. Horizontal polarization shows greater immunity to the shadowing, despite an insignificant impact on the fast fading compared to the vertical polarization.

Nevertheless, overall, fast fading has a major effect on the signal, as Figure 8 shows. This occurs when the coherence time of the channel is small relatively to the delay requirement of the application. In this case, the amplitude and phase change imposed by the channel vary considerably over the period of use.
TABLE 3: Fast-fading modeled with a Rician distribution at the vertical and horizontal polarization directions.

\begin{tabular}{lcc}
\hline & Vertical & Horizontal \\
\hline$K$ factor measurements & $2.62 \mathrm{~dB}$ & $2.62 \mathrm{~dB}$ \\
$K$ factor simulation & $2.79 \mathrm{~dB}$ & $2.62 \mathrm{~dB}$ \\
\hline Std measurements & 0.55 & 0.59 \\
Std simulation & 0.52 & 0.61 \\
\hline
\end{tabular}

Regarding the $K$ factor, the three scenarios present similar results. The relation between $\operatorname{LoS}$ and the multipath component is very low. This is because, as it was described in previous sections, the waveguide effect allows the propagation of a high number of modes at this frequency. As a result, the received signal is essential for not only the LoS component, but also the sum of all the reflected signals along the tunnel.

4.2. Wideband Analysis. This RT based simulator is used to acquire PDPs and AoAs whose information is helpful to understand the wideband channel. In this particular case, the data obtained from the measurements in narrowband are used to parametrize the needed values in the simulator in the wideband analysis.

4.2.1. Power-Delay Profile. The PDPs, in Figure 9(a), reveal that the energy is almost equally divided between LoS and the reflections since the power of these rays has slightly lower power than the one coming from the direct path. This is fully coherent with $K$ factor resulting from the Rician distribution obtained in Section 4.1.2.

Besides, there is a clear decrease in the delay spread when the distance between $\mathrm{Tx}$ and $\mathrm{Rx}$ raises. This is connected with the great attenuation of the higher modes along the tunnel. 



(b) Evolution of AoA

FIGURE 9: Simulation results for (a) PDP and (b) AoA along the distance.

In short distances, as there is an extensive spread of the pulse, signals may be damaged by intersymbol interference.

4.2.2. Angle of Arrival. The AoA, in Figure 9(b), shows also the decrease in the delay spread as Rx moves away from Tx. There is no ray coming from the negative axis of $\phi$ since the horn antenna is directive and the front-to-back ratio is very high, as Figure 5 shows. Besides, all the rays come from the right side of the $\mathrm{Rx}$, which is reasonable as there is misalignment of the antennas imposed by the set-up itself.

On the other side, when the antennas are close, most of the signals arrive from the back side of the antenna, but, when the antennas are sufficiently separated, the different rays are reduced until all the energy is fully concentrated in a single ray.
Considering the shape of the PDP obtained from simulations and the AoA of the rays, it can be assumed that the Doppler spectrum follows an asymmetric Jakes type [23]. It will change from moderate to strong asymmetry when the distance to the transmitter increases, according to [24]. The maximum Doppler deviation for this frequency, considering a $120 \mathrm{~km} / \mathrm{h}$ speed of trains, would be $\pm 9.68 \mathrm{kHz}$.

\section{Conclusion}

In this paper, a narrowband and a wideband study have been performed for $\mathrm{mmW}$ band propagation in subway tunnels on metropolitan railways.

The narrowband analysis takes into account several approaches: measurements, simulations, and a modal propagation model. By combining these techniques, an accurate 
path loss model is obtained. It is clear that there is a considerable lower loss exponent in comparison to the Friis formula since the measurements show a loss exponent of 1.3 in the tunnel. Regarding the simulations, although some limitations have been found when the maximum reflections of each ray are not enough, this inaccuracy is gradually reduced in accordance with the distance as the modes are becoming weaker and, as a result, fewer rays are necessary to represent more precisely the path loss.

The wideband analysis, based on the PDP and the AoA, reveals that the delay spread decreases as Rx moves away from Tx. This is an important result which, once again, highlights that higher modes extinguish faster than the lower ones. For that reason, the behavior of wideband channel improves with the distance to the transmitter.

Finally, an important remark is that there is the absence of published measurement on propagation at $\mathrm{mmW}$ frequencies in real subway tunnels, and this paper validates the possibility of using $\mathrm{mmW}$ frequency bands for communications in metropolitan railway tunnels.

\section{Conflicts of Interest}

The authors declare that there are no conflicts of interest regarding the publication of this paper.

\section{Acknowledgments}

This work was developed under the framework of ENABLING 5G TEC2014-55735-C3-2-R funded by the Spanish Ministry of Economy and Competitiveness and also funded by the Chinese Strategic International Cooperative Project of National key R\&D Plan, 2016YFE0200200.

\section{References}

[1] S. Chen, J. Hu, Y. Shi et al., "Vehicle-to-everything (v2x) services supported by LTE-based systems and 5G," IEEE Communications Standards Magazine, vol. 1, no. 2, pp. 70-76, 2017.

[2] J. Moreno, J. Riera, L. De Haro, and C. Rodriguez, "A survey on future railway radio communications services: challenges and opportunities," IEEE Communications Magazine, vol. 53, no. 10, pp. 62-68, 2015.

[3] A. Gonzalez-Plaza, J. Moreno, I. Val et al., "5G communications in high speed and metropolitan railways," in Proceedings of the 11th European Conference on Antennas and Propagation, EUCAP 2017, pp. 658-660, France, March 2017.

[4] L. Zhang, A. Gonzalez-Plaza, J. R. Fernandez et al., "Propagation measurements and modelling inside trains at $900 \mathrm{MHz}$, 2.4 and 5.8GHz," in Proceedings of the 11th European Conference on Antennas and Propagation, EUCAP 2017, pp. 2895-2898, France, March 2017.

[5] A. Arriola, P. M. Rodriguez, I. Val et al., "Characterization of an Outdoor-to-Indoor wireless link in metro environments at 2.6 $\mathrm{GHz}$," in Proceedings of the 15th International Conference on ITS Telecommunications, ITST 2017, Poland, May 2017.

[6] T. Rosowski, "5G spectrum scenarios, requirements and technical aspects for bands above $6 \mathrm{GHz}, 2016$," https://metis-ii.5gppp.eu/wp-content/uploads/deliverables/METIS-II_D3.1_V1.0 .pdf.
[7] J. Barrett, "Global mobile Suppliers Association, 2017," https:// gsacom.com/5g-spectrum-bands/.

[8] X. Yang and Y. Lu, "Propagation characteristics of millimeter wave in circular tunnels," in Proceedings of the 20067 th International Symposium on Antennas, Propagation \& EM Theory, pp. 1-5, Guilin, China, October 2006.

[9] S.-W. Choi, H.-S. Chung, D.-S. Cho et al., "Performance evaluation of millimeter-wave-based communication system in tunnels," in Proceedings of the IEEE Globecom Workshops, GC Wkshps 2015, USA, December 2015.

[10] W. Hui, Z. Guoxin, and J. Minghua, "The measurements and simulations of millimeter wave propagation at $38 \mathrm{ghz}$ in circular subway tunnels," in Proceedings of the 2008 China-Japan Joint Microwave Conference, CJMW 2008, pp. 51-54, China, September 2008.

[11] N. Prediger and A. Plattner, "Propagation measurements at 60 $\mathrm{GHz}$ in railroad tunnels," in Proceedings of the IEEE/MTT-S International Microwave Symposium - MTT '94, pp. 1085-1087, San Diego, CA, USA.

[12] J. Antes, F. Boes, T. Messinger et al., "Multi-gigabit millimeterwave wireless communication in realistic transmission environments," IEEE Transactions on Terahertz Science and Technology, vol. 5, no. 6, pp. 1078-1087, 2015.

[13] C. Zhou, "Ray tracing and modal methods for modeling radio propagation in tunnels with rough walls," IEEE Transactions on Antennas and Propagation, vol. 65, no. 5, pp. 2624-2634, 2017.

[14] C. Briso-Rodriguez, J. M. Cruz, and J. I. Alonso, "Measurements and modeling of distributed antenna systems in railway tunnels," IEEE Transactions on Vehicular Technology, vol. 56, no. 5, pp. 2870-2879, 2007.

[15] C.-S. Zhang and L.-F. Guo, "Research on propagation characteristics of electromagnetic wave in tunnels with arbitrary cross sections," in Proceedings of the 2010 2nd International Conference on Future Computer and Communication, ICFCC 2010, pp. V122-V125, China, May 2010.

[16] C. Zhou and R. Jacksha, "Modeling and measurement of radio propagation in tunnel environments," IEEE Antennas and Wireless Propagation Letters, vol. 16, pp. 141-144, 2017.

[17] M. Jia and J. Zhao, "A novel method to predict the wideband characteristics of MMW propagation in tunnels," in Proceedings of the 7th International Conference on Wireless Communications, Networking and Mobile Computing, WiCOM 2011, China, September 2011.

[18] I. Val, A. Arriola, P. M. Rodriguez et al., "Wireless channel measurements and modeling for TCMS communications in metro environments," in Proceedings of the 11th European Conference on Antennas and Propagation, EUCAP 2017, pp. 108112, France, March 2017.

[19] Vector Telecom, "A global supplier of microwave components, assemblies and antenna systems, 2017," http://www .vectortele.com/.

[20] K. Guan, B. Ai, Z. Zhong et al., "Measurements and analysis of large-scale fading characteristics in curved subway tunnels at $920 \mathrm{MHz}, 2400 \mathrm{MHz}$, and $5705 \mathrm{MHz}$," IEEE Transactions on Intelligent Transportation Systems, vol. 16, no. 5, pp. 2393-2405, 2015.

[21] A. Hrovat, G. Kandus, and T. Javornik, "A survey of radio propagation modeling for tunnels," IEEE Communications Surveys \& Tutorials, vol. 16, no. 2, pp. 658-669, 2014.

[22] S. Bashir, "Effect of antenna position and polarization on UWB propagation channel in underground mines and tunnels," IEEE 
Transactions on Antennas and Propagation, vol. 62, no. 9, pp. 4771-4779, 2014.

[23] X. Zhao, J. Kivinen, P. Vainikainen, and K. Skog, "Characterization of doppler spectra for mobile communications at $5.3 \mathrm{GHz}$," IEEE Transactions on Vehicular Technology, vol. 52, no. 1, pp. 1423, 2003.

[24] X. Chen, Y. Pan, Y. Wu, and G. Zheng, "Research on Doppler spread of multipath channel in subway tunnel," in Proceedings of the 2014 IEEE International Conference on Communication Problem-Solving, ICCP 2014, pp. 56-59, China, December 2014. 




\section{Enfincering}
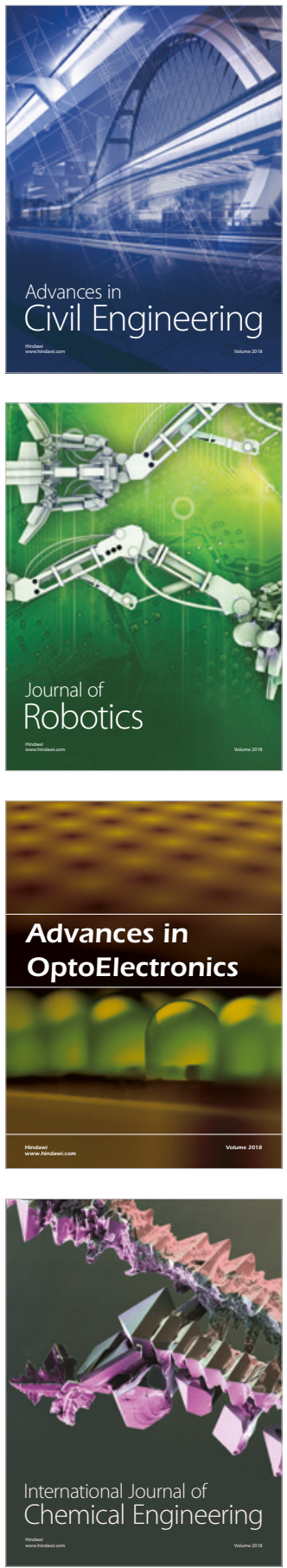

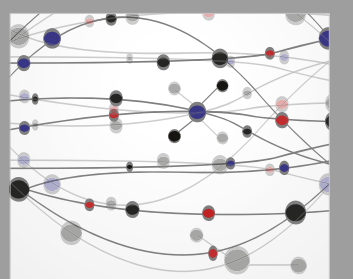

\section{Rotating \\ Machinery}

The Scientific World Journal



Submit your manuscripts at

www.hindawi.com
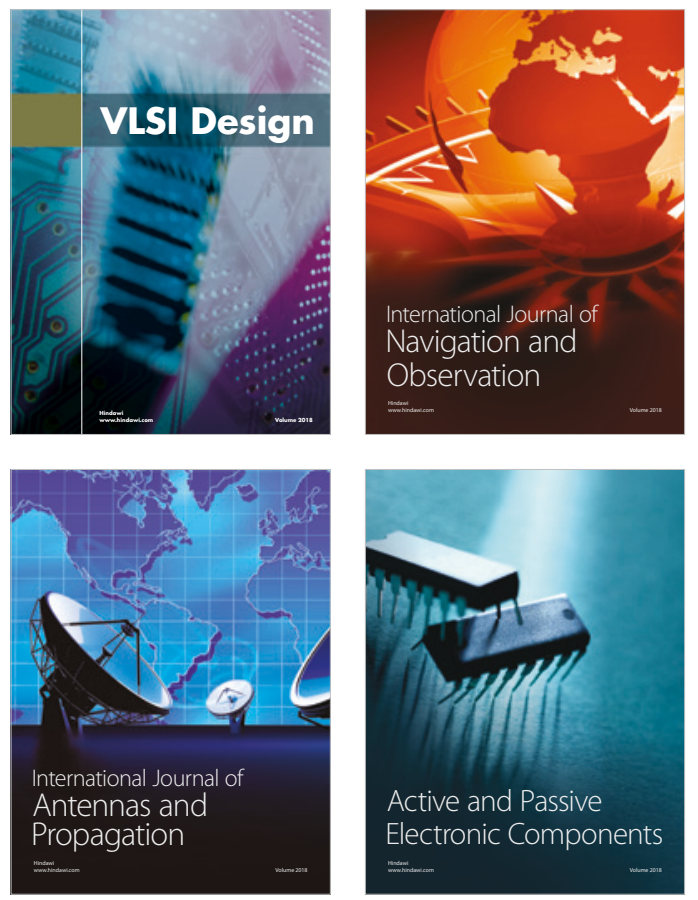
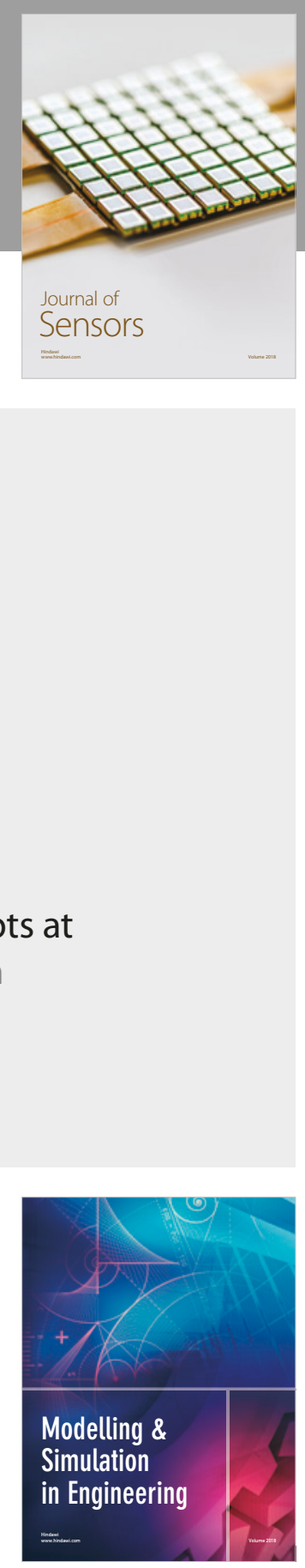

\section{Advances \\ Multimedia}
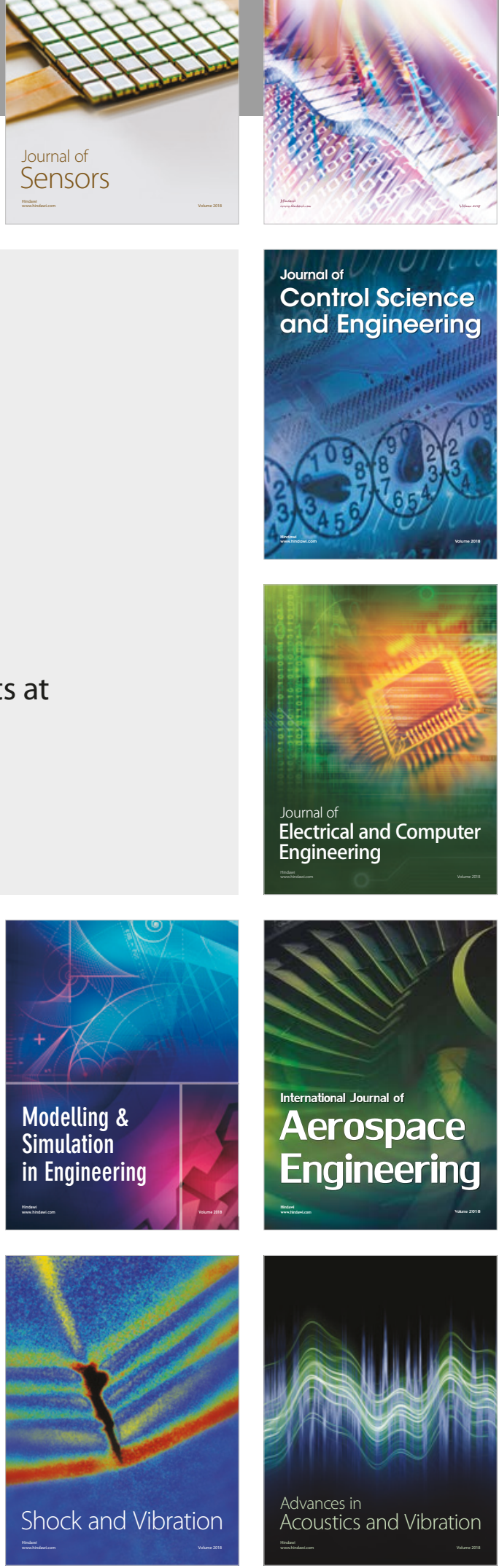